\title{
Heat Health Prevention Measures and Adaptation in Older Populations-A Systematic Review
}

\author{
An $\mathrm{Vu}^{*}$, Shannon Rutherford * and Dung Phung \\ School of Medicine, Gold Coast Campus, Griffith University, QLD 4222, Australia; d.phung@griffith.edu.au \\ * Correspondence: an.vu2@alumni.griffithuni.edu.au (A.V.); s.rutherford@griffith.edu.au (S.R.)
}

Received: 4 October 2019; Accepted: 6 November 2019; Published: 8 November 2019

check for updates

\begin{abstract}
The population of older people is increasing at a rapid rate, with those 80 years and older set to triple by 2050. This systematic review aimed to examine older people's perceptions and behaviours against existing heatwaves prevention measures and systematically categorize and analyse those measures using the Ottawa charter for health promotion framework. Peer-reviewed published literature between 22nd September 2006 and 24th April 2018 was retrieved, according to the PRISMA guidelines, from five different databases. Eighteen articles were finally included. There is a lack of published studies from developing countries. Results were categorized and analysed using the Ottawa charter five action areas. Mitigation strategies from current heat action plans are discussed and gaps are highlighted. A lack of systematic evaluation of heat action plans efficacy was identified. Older people are not demonstrating all recommended preventative measures during heatwaves. Support personnel and health professionals are not being pro-active enough in facilitating prevention of adverse effects from heatwaves. Governments are beginning to implement policy changes, but other recommended support measures outlined in the Ottawa charter are still lacking, and hence require further action. Linkage between specific components of heat action plans and outcomes cannot be ascertained; therefore, more systematic evaluation is needed.
\end{abstract}

Keywords: heatwaves; older people; vulnerable population; heat action plans; prevention; adaptation; Ottawa charter

\section{Introduction}

The intergovernmental panel on climate change [IPCC] has reported with medium to high confidence that longer lasting and more intense increases in ambient temperature or heatwaves will continue to cause adverse impacts on human health across the globe [1]. Heatwaves, as a consequence of human-induced climate change are widely acknowledged amongst the scientific community as a real and present threat to human health [1]. While the detrimental impacts of climate change continue to transcend the trajectory of time, natural ecosystems and human species have limited capacity to adapt to such changes [2]. Historical examples occurred in Chicago in 1995, across Europe in 2003, Australia in 2009, and Russia in 2010, resulting in a total of over 82,000 excess deaths from heatwaves with older people over-represented [3-8]. Consequently, increasing morbidity and mortality from extreme temperatures is becoming a major public health concern for communities worldwide, especially amongst vulnerable groups [9]. 
Studies on heat-related morbidity and mortality have consistently identified subgroups within the population that are more vulnerable to heat, with a disproportionate percentage of older people to be influenced by these extreme temperature changes $[10,11]$. Much is known of the physiological effects of heat stress, with many studies linking extreme heat to increasing morbidity and mortality [12]. Older people have less capacity to adapt to heat stress due to natural physiological decline and impaired thermoregulatory functions [13]. In addition, older people also tend to suffer more from chronic diseases compared with younger age groups [14].

Research has shown that those suffering from chronic diseases, such as diabetes and cardiovascular or respiratory illnesses, have even less capacity to adapt to sudden fluctuations in temperature during a heatwave [13]. Oudin Åström et al. [10] reported a strong correlation between increasing mortality rates amongst older people already suffering from cardiorespiratory illnesses. Moreover, Yu et al. [15] have pinpointed a relationship of $2-5 \%$ increase in all-cause mortality per $1{ }^{\circ} \mathrm{C}$ increase in temperature. Medication dependency further compounds the adverse health risks from extreme heat [16]. This scenario applies to older people worldwide [10,17].

Longer life expectancies and falling fertility rates means that the global population of older people continues to grow at a steady rate, with those 60 years and over surpassing all groups under 60 , estimated at 2.1 billion by 2050 and 3.1 billion by 2100 [18]. In 2018, those aged 65 and over outnumbered children under five years of age. The population of older people aged 80 years and over is expected to triple to 426 million by 2050 from 143 million currently [19].

Age, coupled with pre-existing illnesses and several other extrinsic and intrinsic factors, contributes to the overall vulnerability of this population [20]. Factors such as low socio-economic status, ethnicity, gender, lack of education, living alone, or infrequent social outings have all been linked to higher risks of heat-related deaths $[3,21-25]$. Therefore, it is paramount that measures are in place to mitigate the effects of heat vulnerability and the resultant burden of disease in this population.

This systematic review aims to contribute to the current body of research on factors influencing older people's adaptive behavior and current preventative strategies during extreme heat events, with two objectives: (i) investigate older people's thoughts and behaviors in relation to current mitigation measures and their evaluations, in preventing adverse outcomes of heatwaves, and (ii) systematically categorize and analyze those findings by using a health promotion framework.

\section{Ottawa Charter for Health Promotion Framework}

The gradient of health to illness corresponds to a social gradient of high to low socio-economic status [26]. Ultimately, the social determinants of health are the conditions and environment in which people are born, grow, live, work, love, and age [27]. This systematic review defines 'heat prevention, adaptation or mitigation measures' as actions taken by the individuals, as well as local communities and federal agencies as specified by regional legislations and policies, hence the use of the Ottawa charter as a framework. It is indeed essential to examine older people as target populations, but also their thoughts and behaviors so as to inform implementation and/or refinement of policies and procedures catered for such populations.

On the basis of this, it is appropriate to utilize the Ottawa charter as a framework to identify inequities and inequalities influencing the health outcomes of older populations in the face of climate change-induced extreme heat events. Initially proposed as a means to promote individuals' control and improvement of their health, adoption of the framework is correlated with positive outcomes of health promotion programs [28]. This framework comprises of five areas for action: 1 . Build healthy public policy, 2. Create supportive environments, 3. Strengthen community actions, 4 . Develop personal skills, and 5. Reorient health services. 
Build healthy public policy: Optimal population health begins with responsible management [27]. Therefore, governments' fiscal policies and legislation require multi-sectoral input and appropriate prioritization while taking into account social policies to ensure greater equity. This leads to healthier environments in which goods and services are accessible to all. Subsequently, barriers to adopting healthy public policies are lifted, ensuring easier access to health even from non-health sectors. Thus, the healthier choice becomes the natural choice for both consumers and policy makers alike [27].

Create supportive environments: The socio-ecological model of health highlights the interrelatedness and complexities of individuals within societies and their inseparable connections to their surroundings [27]. On the basis of this advances the notion of sustainability and conservation of our natural resources. Accordingly, compromises must be made to ensure harmonious integration of the built environments while maintaining the integrity of natural surroundings. Only by conserving finite natural resources in rapidly changing environments can we facilitate safe and stimulating living and working conditions that are satisfying, enjoyable, and conducive to health [27].

Strengthen community actions: Empowering communities to take charge of their own fates through collective decision making, planning, and execution of contextually relevant priorities would ensure healthier lives as a whole [27]. Consequently, it is of paramount importance that those communities are given the means to help themselves as well as providing support for others, which in turn facilitates the growth of social capital and strengthens the support networks within, benefiting them both individually and collectively [27].

Develop personal skills: Individuals require a repertoire of knowledge and skills to draw upon in selecting the best choices conducive to and in control of their health [27]. These choices take into account the individuals' personal circumstances, whether physical, psychological, or socioeconomic, in relation to their immediate environments. Therefore, it is essential that people are provided with appropriate information to enhance their health literacy and life skills through various formal and informal institutions, including educational, professional, commercial, and voluntary bodies [27].

Reorient health services: In this globalized world it is crucial that healthcare systems deliver culturally sensitive and contextually sound services [27]. Those systems must also consider the needs of each individual as a whole in the pursuit of community health. Subsequently, those learning healthcare systems require a concerted effort that transcends various socio-political institutions as well as private and public sectors, resulting in a multi-sectoral wholesome collaboration that is beyond purely clinical and curative services $[28,29]$.

\section{Methods}

This review followed the preferred reporting items for systematic reviews and meta-analyses (PRISMA) checklist for systematic reviews [30].

\subsection{Specifications for Systematic Review}

The research question and evidence collection was formulated according to four elements: population, intervention, comparison and outcomes (PICO) (see Table 1).

Table 1. The PICO strategy.

\begin{tabular}{cc}
\hline Parameters & Details \\
\hline Population & Older people 65 years and over \\
\hline Intervention & Heat prevention, adaptation or mitigation measures \\
\hline Comparison & No prevention measures \\
\hline Outcomes & Any observable/measurable effects of heat related mortality or morbidity \\
\hline
\end{tabular}




\subsection{Search Strategy}

Online databases were searched, including the cumulative index of nursing and allied health literature (CINHAL), medical literature analysis and retrieval system online (MEDLINE), PubMed, excerpta medica database (EMBASE), and web of science. All studies since inception to July 2019 were searched using Boolean logic and key words as follows: (Heat OR heat-related OR "high temperatures" OR heatwaves) AND (Elderly OR older people OR seniors OR "vulnerable populations" OR "vulnerable groups") AND (Management OR "preventative measures" OR prevention OR solutions OR mitigation OR adaptation OR intervention OR evaluation) NOT (Occupation OR work). Duplicates were removed in EndNote library and the three-tier approach was utilized-article titles, abstracts, and full-texts were screened against inclusion and exclusion criteria.

\subsection{Inclusion and Exclusion Criteria}

Peer-reviewed, full-text, qualitative, and/or quantitative studies in English of any design/methodology were considered. Those investigating or evaluating vulnerability and/or adaptability of older people 65 years and older living independently, against a heat action plan (HAP) or preventative measures, were included in this study. Conference abstracts or reviews/editorials, non-English, full-text articles, and grey literature were excluded.

\subsection{Quality Assessment}

Due to heterogeneity of methodology and designs of included studies, it was not possible to apply stringent guidelines in quality assessment.

\subsection{Data Analysis and Synthesis}

Included studies were analyzed and data were extracted onto a spreadsheet under key headings author/year, country, objectives, subjects, study design, intervention/factors, outcomes, and recommendations. A narrative approach was undertaken to synthesize the results since a quantitative meta-analysis was impossible to perform, due to studies' heterogeneity in methodology, designs, definitions of exposure, and outcomes. To structure the analysis, this review adopted a novel approach by grouping the findings into categories and guided discussion using the Ottawa charter for health promotion framework [27].

\section{Results}

A total of 7534 articles were found from electronic database searching. These were imported into the EndNote library and duplicates were removed, leaving 7133 articles. Title screening eliminated a further 6513 articles. Altogether, 620 articles underwent further abstract screening and 30 articles were selected for full-text screening. Of these, one study examined nursing home residents only [31]; one study did not fulfil the minimum age limit of 65 [32]; one article discussed heat susceptibility and health promotion in general but did not conduct any studies or evaluation [33]; one study discussed health promotion but not related to heat health [34]; three studies assessed mortality/morbidity rates related to heat but not against any components of heat action plans [35-37]; five articles were not assessing heat health and/or older people specifically [38-42] —all twelve were excluded. A total of 18 articles remained for analysis (See Figure 1). 

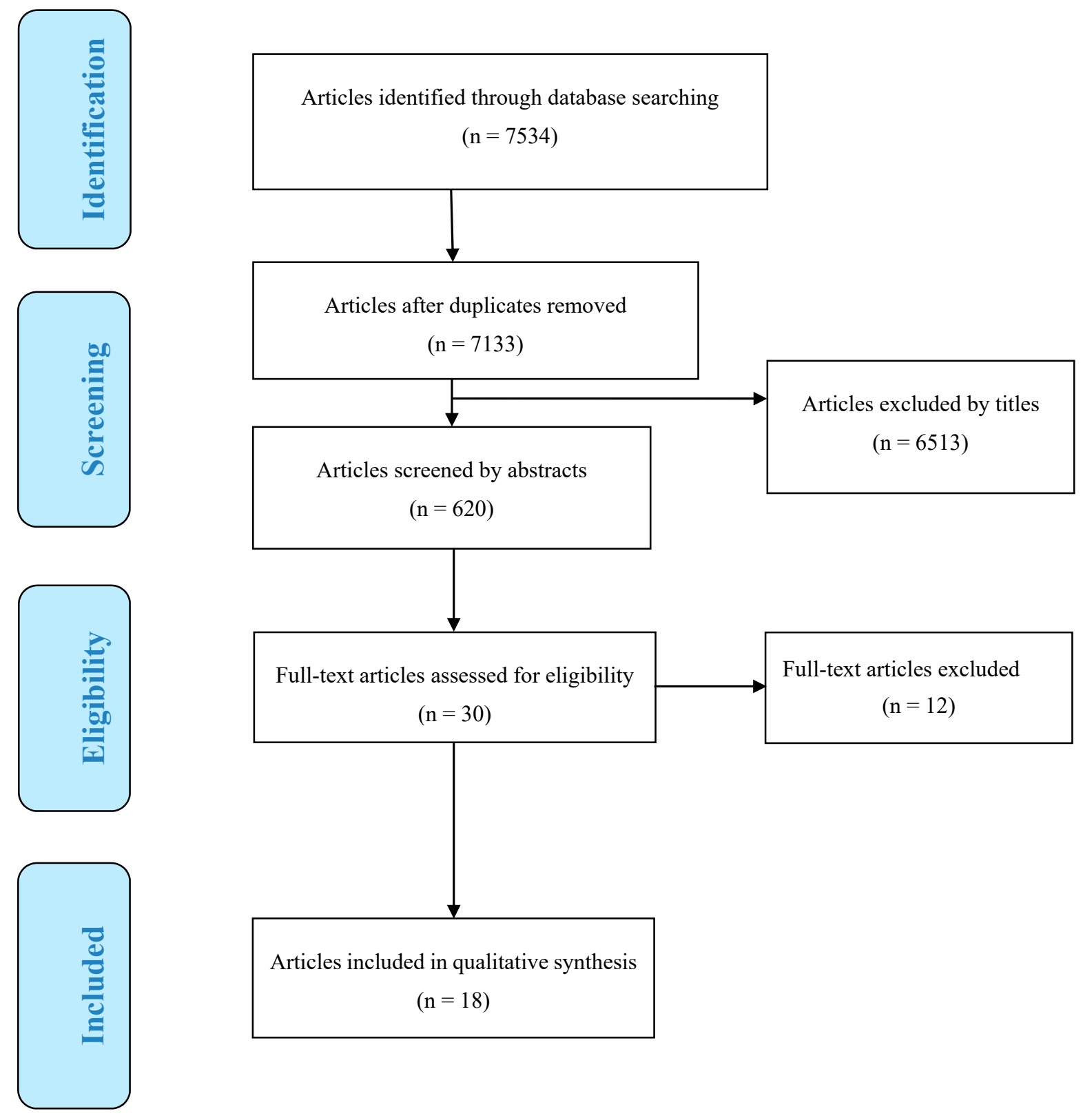

Figure 1. PRISMA flow diagram for literature search.

\subsection{Study Characteristics}

Results of selected studies have been summarized in Table 2. Date of publication ranged from 22nd September 2006 to 24th April 2018. All studies were in developed countries-seven from Australia [43-49], three from Italy [50-52], three from America/Canada [53-55], three from the UK [56-58], one from Germany [59], and one from Japan [60].

Various designs and methods of analysis were employed. There were two randomized controlled trials (RCTs) [49,60], ten cross-sectional surveys via telephone or online, and/or face-to-face semi-structured interviews or focus groups [43-47,54,56-59], one logbook/diary survey [55], two quasi-experimental retrospective studies [50,53], and the rest were meta-analyses investigating mortality/morbidity rates against maximum apparent temperatures [51], time-series random effect multivariate evaluating older people's daily mortality rates pre- and post-introduction of the 'national heat health prevention program' [52] and incidence rate ratios of pre- and post-exposure to a prevention program [48]. 


\subsection{Heat Warning Systems and Heat Action Plans}

The studies gathered described an array of heat warning systems (HWSs) within countries and across the world. All 18 studies outlined different components of heat action plans (HAPs); albeit, some described and analyzed them in more detail than others (e.g., [50,53,54]). In places with established HWSs and HAPs, an array of different meteorological methods can be found in measuring temperatures and the resultant triggering thresholds for public warnings (e.g., [50-54]. It seems that geographical and climatic differences between regions have influenced the methodology and implementation of warning systems. For example, Philadelphia's HWS (Pennsylvania, USA) is the oldest, compared with Dayton (Ohio, USA), Phoenix (Arizona, USA), and Toronto (Ontario, Canada) [54]. Instrument-wise, Michelozzi et al. [51] described two different models for estimating daily mortality using maximum apparent temperature and oppressive air mass approaches. Moreover, Sheridan [54] revealed that while Dayton issues alerts if one to two deaths are expected, Philadelphia only sends out alerts when four or more deaths are expected.

Monumental heatwaves in 2003 prompted many countries in Europe to implement both formal and informal processes to combat future occurrences. Abrahamson et al. [57] have included a revised 2004 HAP from England's department of health which outlines protocols for national, regional, and local levels, as well as most-at-risk subpopulations-especially females $>75$, those with chronic and mental health illnesses, and people living or working in overexposed areas. Michelozzi et al. [51] provided a complete overview of Italy's department for civil protection and the ministry of health national HWSs and HAPs, including different methodologies to arrive at implementation for each city, such as local bulletin networks, local registries for subgroups, and a real-time surveillance system. The Italian national prevention program has been operational since 2004 and covers $93 \%$ of residents 65 years and over in 34 cities [51,52]. Liotta et al.'s study [50] focused more at a local measure in Rome, evaluating the long live the elderly (LLE) program, designed to counteract social isolation in urban residents $>75$ years of age. In Germany, the German meteorological service issues warnings when temperature exceeds $32{ }^{\circ} \mathrm{C}$ on two consecutive days [59] and informal procedures have been in place for over a decade until 2017, when more formal procedures were implemented [61].

Elsewhere, the Canadian Montreal public health department (PHD) implemented a HAP in 2004, as described by [53]. Examples provided includes activation of alerts by the public health department, through to local activities such as provision of light meals and bottled water, to increasing frequency of phone calls and home visits, to vulnerable people in the community. In the USA and Canada, [54] there was no formal found in HAP in Phoenix, an informal HAP in Dayton, and more formalized HAPs in Toronto and Philadelphia. The Combined Health District of Montgomery County is responsible for heat alerts in Dayton, which coordinates with over 150 local governmental and non-governmental agencies to disseminate heat warnings, advice on how to prevent heat stress, as well as operating hotlines and a "buddy system" in which older people are checked on by volunteers and neighbors. Philadelphia has the most extensive and oldest HWS, dating back to 1991. The division of health promotion coordinates education campaigns and heat-health advisories linking thousands of volunteers and organizations, such as the Philadelphia corporation for the aging to at-risk individuals, via media publications and hotlines. The department of public health is responsible for provision of increased staffing, utilities, and services, such as cooling centers, as required. In Toronto, Environment Canada coordinates with Toronto public health to issue heat alerts and disseminate information to a thousand volunteers and agencies [54]. These local groups are responsible for facilitating hotlines, healthcare professionals, opening cooling centers and home visits, as well as street patrols to deliver bottled water. Despite no formal HAP in Phoenix, the local national weather service agency provides the most detailed advisories that local media can disseminate on a voluntary basis.

Australia currently does not have a formalized national HAP. The government of South Australian capital city Adelaide adopted a more formal HWS only after the damaging 2009 heatwave. The Australian Victorian state government has its own heatwave warning protocols $[45,48,49]$. In Adelaide, the state emergency service coordinates with the bureau of meteorology to activate 
heatwave alerts and disseminate prevention advice via media announcements. Pre-determined at-risk individuals are registered by family, friends, and treating GPs with daily phone contacts and home visits as required by NGOs, such as the Australian red cross. The Victorian HAP referenced by Hansen et al. [44] is a revised version of the 2009-2010 frameworks, the auditor general's 2014 account heatwave management: reducing the risk to public health and the state heat plan 2014 combined [62]. It sets forth protocols for pre-season, during, and after a heatwave to evaluate the effectiveness of activities undertaken. The department of health and human services chief health officer coordinates with the emergency management commissioner and the bureau of meteorology to disseminate alerts and detailed advice to the public.

The Japanese HWS was developed in 2006 under the management of the ministry of the environment and the ministry of health, labor, and welfare [60,63]. Local prevention activities rely heavily on volunteer groups known as 'Minsei-iin' or welfare commissioner committees [60].

\subsection{Risk Factors Awareness, Perception of Vulnerability, and Protective Behaviors}

Factors such as age, low socio-economic status, living alone, lack of social outings, use of mobility aids, comorbidities, and use of medications have all been identified as risk factors during heatwaves $[43,49,50,53,55]$. In general, the majority of subjects were aware of physical health risks but less so of social risks [58]. Healthcare professionals displayed the most awareness of their patients' risks while community workers displayed less, with crucial knowledge gaps identified regarding specific mechanisms of thermoregulation and safe use of electric fans [45,46,60]. Notwithstanding knowledge levels, in four studies from Australia, UK, and Germany, frontline healthcare professionals appeared to be too complacent and not proactive in seeking out the most vulnerable older people during heatwaves $[43,46,56,59]$.

Despite awareness of danger, many older people surveyed in Australia, UK, and USA/Canada did not feel they were susceptible to heat-related illness $[54,57,58]$. Many respondents were not concerned upon hearing heatwave warnings, with $74 \%$ reporting that they should not have to stop daily activities, keeping most of their usual appointments, and would still exercise despite the heat [47]. In addition, those nominated by older people as advisors/assistance were not proactive in reaching out to them prior to heat events, but rather waiting for them to call when already distressed, citing reluctance to impingement on their independence [58].

In regard to protective behaviors, respondents called upon 'common sense' [56,57]. Most displayed adaptive behaviors such as wearing loose clothing, taking showers, and drinking more water $[45,48]$. However, the full range of protective behaviors was not demonstrated, as evidenced in White-Newsome et al.'s study in Detroit, USA [55]. In instances where bottled water was delivered with heat-health messages, increased water intake was noted, and alternative cooling methods employed [60]. Indoor temperature influenced behavior up to a threshold with increasing temperature lowering the likelihood of people leaving their home or adopting alternative cooling methods, such as seeking cooling centers [55]. At least a third of respondents expressed concerns regarding costs of running air conditioners [49,54].

\subsection{Effectiveness and Limitations of Heat Action Plans}

The success in mortality and/or morbidity rates reduction and improvement in adaptive behaviors have been attributed to introduction of HAPs [48,51-53]. However, the results show that while in general most subjects were aware of heat warnings and advice, many were also confused as to what to do exactly [54]. Some studies have shown evaluation of various components of respective regional HAPs, but direct causal relationships between individual components of HAPs and quantitatively measurable outcomes/behaviors have not been established [48-50,52,58,60]. 
Table 2. Summary of selected studies.

\begin{tabular}{|c|c|c|c|c|c|c|c|}
\hline Author and Year & Country & Objectives & Subjects & Study Design & Intervention or Factors & Outcomes & Recommendations \\
\hline $\begin{array}{l}\text { Hansen et al. } \\
2011[43]\end{array}$ & & $\begin{array}{c}\text { Investigate } \\
\text { heat-susceptibility in } \\
\text { older people and } \\
\text { perceived adaptation } \\
\text { barriers during } \\
\text { heatwaves in Adelaide }\end{array}$ & $\begin{array}{l}\quad \mathrm{n}=35 \\
\text { four groups of } \\
\text { health providers, } \\
\text { managers and } \\
\text { policy makers }\end{array}$ & $\begin{array}{c}\text { Telephone } \\
\text { interviews and } \\
\text { focus groups }\end{array}$ & $\begin{array}{l}\text { Questioning respondents } \\
\text { knowledge of risks to } \\
\text { older people and barriers } \\
\text { to adaptive behaviours }\end{array}$ & $\begin{array}{c}\text { Respondents identified } \\
\text { physiological (poor health, chronic } \\
\text { conditions, functional disabilities), } \\
\text { socioeconomic issues (costs } \\
\text { associated with running } \\
\text { air-conditioners), psychological } \\
\text { issues (anxieties, cognitive } \\
\text { dysfunction), and barriers/enablers } \\
\text { to adaptive strategies }\end{array}$ & $\begin{array}{l}\text { Clear instructions on } \\
\text { operation of } \\
\text { air-conditioners } \\
\text { Energy rebates for older } \\
\text { people } \\
\text { Specific strategies for } \\
\text { specific medical } \\
\text { conditions }\end{array}$ \\
\hline $\begin{array}{l}\text { Hansen et al. } \\
2015 \text { [44] }\end{array}$ & & $\begin{array}{l}\text { Investigate prevention } \\
\text { behaviours (PB) of } \\
\text { independently living } \\
\text { residents in South } \\
\text { Australia and Victoria }\end{array}$ & $\begin{aligned} \mathrm{n} & =1000 \\
& \geq 65\end{aligned}$ & $\begin{array}{l}\text { Cross-sectional } \\
\text { Telephone survey }\end{array}$ & $\begin{array}{c}\text { Demographics, social } \\
\text { contacts, self-evaluated } \\
\text { health status, coping } \\
\text { strategies, medications, } \\
\text { air conditioning, and heat } \\
\text { warnings }\end{array}$ & $\begin{array}{l}\text { Most demonstrated PB; More heat } \\
\text { warnings recall and AC in South } \\
\text { Australia vs. Victoria; Female sex, } \\
\text { chronic illness sufferers reported } \\
\text { increased morbidity }\end{array}$ & $\begin{array}{l}\text { Review current policies } \\
\text { Disseminate heat } \\
\text { warnings via media and } \\
\text { SMS }\end{array}$ \\
\hline $\begin{array}{l}\text { Ibrahim et al. } \\
2012[45]\end{array}$ & Australia & $\begin{array}{l}\text { Investigate healthcare } \\
\text { providers current } \\
\text { practices to care for } \\
\text { older people living } \\
\text { independently in } \\
\text { Victoria }\end{array}$ & $\mathrm{n}=327$ Six groups & $\begin{array}{l}\text { Cross-sectional } \\
\text { electronic survey }\end{array}$ & $\begin{array}{c}32 \text { questions - } \\
\text { demographic, } \\
\text { professional } \\
\text { characteristics, heatwave } \\
\text { impacts, heat health } \\
\text { knowledge, current } \\
\text { practices to treat } \\
\text { heat-related illness }\end{array}$ & $\begin{array}{l}\text { Most aware of danger to older } \\
\text { people; Gaps in knowledge: } \\
\text { thermo-regulation, electric fans use } \\
\text { and most critical time to offer help; } \\
\text { Few emergency plans in place; } \\
\text { Reactive and opportunistic in } \\
\text { practices }\end{array}$ & $\begin{array}{l}\text { Emergency response } \\
\text { plans needed } \\
\text { Improvement required in } \\
\text { knowledge Call for a } \\
\text { more proactive approach }\end{array}$ \\
\hline $\begin{array}{l}\text { McInnes et al. } \\
2010[46]\end{array}$ & & $\begin{array}{c}\text { Investigate roles of } \\
\text { community } \\
\text { organisations and } \\
\text { health providers in } \\
\text { reducing harm to } \\
\text { older people living } \\
\text { independently in } \\
\text { Victoria }\end{array}$ & $\mathrm{n}=12$ Four groups & $\begin{array}{l}\text { Cross-sectional } \\
\text { study, face-to-face } \\
\text { and telephone } \\
\text { survey }\end{array}$ & $\begin{array}{l}\text { Semi-structured } \\
\text { interviews exploring their } \\
\text { roles in an heatwave } \\
\text { emergency and issues } \\
\text { such as coordination, } \\
\text { identification of high-risk } \\
\text { persons and } \\
\text { training/education }\end{array}$ & $\begin{array}{l}\text { No formal heat action plans } \\
\text { (HAPs); At-risk individuals } \\
\text { identified prior to summer; Good } \\
\text { communication networks available, } \\
\text { potentially able to provide } \\
\text { appropriate care but lacking } \\
\text { coordination and training; Mainly } \\
\text { reactive and opportunistic activities }\end{array}$ & $\begin{array}{c}\text { Need formalised heat } \\
\text { action plans } \\
\text { More proactive strategies } \\
\text { and practices } \\
\text { More resources and } \\
\text { training needed } \\
\text { Develop 'buddy' system } \\
\text { of volunteers }\end{array}$ \\
\hline $\begin{array}{l}\text { Nitschke et al. } \\
2013 \text { [47] }\end{array}$ & & $\begin{array}{l}\text { Investigate resilience, } \\
\text { prevention behaviours, } \\
\text { risk factors and health } \\
\text { outcomes of } \\
\text { independently living } \\
\text { residents in South } \\
\text { Australia }\end{array}$ & $\begin{aligned} \mathrm{n} & =499 \\
& \geq 65\end{aligned}$ & $\begin{array}{l}\text { Cross-sectional } \\
\text { computer assisted } \\
\text { telephone survey }\end{array}$ & $\begin{array}{c}\text { Survey explored } \\
\text { demographics, housing, } \\
\text { social connectedness, } \\
\text { self-reported health status } \\
\text { and vulnerability, heat } \\
\text { health knowledge and } \\
\text { resilience }\end{array}$ & $\begin{array}{l}\text { Majority are resilient; Variety of } \\
\text { prevention behaviours reported; } \\
\text { High medication usage for chronic } \\
\text { diseases, female sex, mobility aids, } \\
\text { chronic diseases, mental health } \\
\text { increased risk and poorer outcomes; } \\
\text { Less social contact for those }<75\end{array}$ & $\begin{array}{c}\text { Targeted intervention } \\
\text { required to address } \\
\text { medication use, } \\
\text { co-morbidities, } \\
\text { knowledge improvemen } \\
\text { and social isolation } \\
\text { Policy development } \\
\text { required }\end{array}$ \\
\hline
\end{tabular}


Table 2. Cont

\begin{tabular}{|c|c|c|c|c|c|c|c|}
\hline Author and Year & Country & Objectives & Subjects & Study Design & Intervention or Factors & Outcomes & Recommendations \\
\hline $\begin{array}{l}\text { Nitschke et al. } \\
\quad 2016[48]\end{array}$ & & $\begin{array}{l}\text { Investigate } \\
\text { effectiveness of } \\
\text { heatwave warning } \\
\text { system in Adelaide }\end{array}$ & $\begin{array}{l}\text { Residents of all } \\
\text { ages }\end{array}$ & $\begin{array}{l}\text { Comparing } \\
\text { morbidity-mortality } \\
\text { data ecological } \\
\text { design }\end{array}$ & $\begin{array}{l}\text { Incidence rate ratios } \\
\text { (IRRs) of daily ambulance } \\
\text { call-outs, emergency } \\
\text { presentations and } \\
\text { mortality data from } 2009 \\
\text { and } 2014 \text { heatwaves } \\
\end{array}$ & $\begin{array}{l}\text { Significant reduction in morbidity } \\
\text { especially emergency presentations } \\
\text { in } 75+\text { group; No reduction in } \\
\text { mortality rate }\end{array}$ & $\begin{array}{l}\text { In-depth assessment of } \\
\text { services provided during } \\
\text { heatwave including reach } \\
\text { and behaviour change } \\
\text { More studies into } \\
\text { mortality risks factors }\end{array}$ \\
\hline $\begin{array}{l}\text { Nitschke et al. } \\
2017 \text { [49] }\end{array}$ & & $\begin{array}{c}\text { Investigate } \\
\text { effectiveness of } \\
\text { targeted information } \\
\text { in preventing adverse } \\
\text { health outcomes } \\
\text { during heatwave }\end{array}$ & $\begin{aligned} \mathrm{n} & =637 \\
& \geq 65\end{aligned}$ & RCT & $\begin{array}{l}\text { Intervention group } \\
\text { provided with specific } \\
\text { instructions on heat } \\
\text { protective measures; } \\
\text { Control group advised to } \\
\text { follow media and seek } \\
\text { own medical assistance as } \\
\text { needed }\end{array}$ & $\begin{array}{l}\text { Higher use of AC, wet cloth on } \\
\text { face/body and significant heat } \\
\text { stress reduction in intervention } \\
\text { group; Control group also } \\
\text { demonstrated protective } \\
\text { behaviours through media }\end{array}$ & $\begin{array}{l}\text { Results generalizable to } \\
\text { other older people } \\
\text { population in SA } \\
\text { Further studies on built } \\
\text { environment thermal } \\
\text { comfort, social services, } \\
\text { GPs active involvement }\end{array}$ \\
\hline $\begin{array}{c}\text { Liotta et al. } 2018 \\
\text { [50] }\end{array}$ & \multirow{3}{*}{ Italy } & $\begin{array}{l}\text { Assess effectiveness of } \\
\text { long live the elderly } \\
\text { (LLE) program in } \\
\text { reducing heat-related } \\
\text { mortality from social } \\
\text { isolation of } \\
\text { independently living } \\
\text { residents }\end{array}$ & $\begin{aligned} \mathrm{n} & =12207 \\
& \geq 75\end{aligned}$ & $\begin{array}{l}\text { Quasi- } \\
\text { experimental } \\
\text { retrospective } \\
\text { cohort study }\end{array}$ & $\begin{array}{l}\text { Intervention group given } \\
\text { social support and all } \\
\text { health needs via both } \\
\text { formal institutions and } \\
\text { volunteers; No extra } \\
\text { support for control group; } \\
\text { Mean property tax } \\
\text { evaluation determined } \\
\text { SES }\end{array}$ & $\begin{array}{l}\text { Mortality rate reduced } 13 \% \text { under } \\
\text { LLE with } 25 \text { deaths averted; LLE } \\
\text { indirectly reduced impact of low } \\
\text { SES and mortality }\end{array}$ & $\begin{array}{l}\text { Routine assessments of } \\
\text { older people and } \\
\text { provision of case-specific } \\
\text { social services could } \\
\text { improve health outcomes } \\
\text { during heatwaves }\end{array}$ \\
\hline $\begin{array}{c}\text { Michelozzi et al. } \\
2010 \text { [51] }\end{array}$ & & $\begin{array}{l}\text { Analyse current } \\
\text { practices and } \\
\text { methodologies of the } \\
\text { Italian national heat } \\
\text { prevention program }\end{array}$ & $\begin{array}{c}93 \% \text { residents } \geq 65 \\
\text { across } 34 \text { cities }\end{array}$ & $\begin{array}{l}\text { Examine dose- } \\
\text { response } \\
\text { relationship } \\
\text { between mortality } \\
\text { and maximum } \\
\text { apparent } \\
\text { temperature } \\
\text { (MAT) }\end{array}$ & $\begin{array}{l}\text { Assessing strengths and } \\
\text { limitations of different } \\
\text { methods to monitor daily } \\
\text { summer mortality in 2008, } \\
2003 \text { and reference period } \\
1995-2002, \text { using Rome } \\
\text { and Milan as examples }\end{array}$ & $\begin{array}{l}\text { Mortality (MAT) differed across } \\
\text { cities; City-specific warning } \\
\text { systems, coordinated central } \\
\text { information network, constant } \\
\text { modulation of preventative } \\
\text { measures major strengths; Specific } \\
\text { prevention programs ensured } \\
\text { timely mitigation measures; } \\
\text { Reduction in mortality rate } \\
\text { attributable to prevention strategies }\end{array}$ & $\begin{array}{l}\text { Implement local registries } \\
\text { to identify vulnerable } \\
\text { individuals - ensures } \\
\text { uniform identification } \\
\text { At-risk individuals } \\
\text { require specific home-care } \\
\text { plans } \\
\text { Further assessment of heat } \\
\text { mitigation plans required }\end{array}$ \\
\hline $\begin{array}{l}\text { Schifano et al. } \\
2012[52]\end{array}$ & & $\begin{array}{l}\text { Investigating } \\
\text { effectiveness of } \\
\text { heatwave prevention } \\
\text { plans post-2003 }\end{array}$ & $\begin{array}{l}\text { Residents } \geq 65 \\
\text { across } 16 \text { cities }\end{array}$ & $\begin{array}{l}\text { Multi-centre time } \\
\text { series (1998-2002) } \\
\text { vs. (2006-2010) } \\
\text { random effect } \\
\text { multi-variate } \\
\text { meta-analysis }\end{array}$ & $\begin{array}{c}\text { Comparing } 16 \\
\text { city-specific daily } \\
\text { mortality rates pre and } \\
\text { post heat prevention } \\
\text { measures, by studying } \\
\text { relationships between } \\
\text { mortality and maximum } \\
\text { apparent temperature }\end{array}$ & $\begin{array}{l}\text { Observable reduction in effects of } \\
\text { high temperature on mortality rates } \\
\text { attributable to mitigation plans }\end{array}$ & $\begin{array}{l}\text { More attention needed at } \\
\text { beginning of summer } \\
\text { when populations yet to } \\
\text { adapt to heat and } \\
\text { prevention activities not } \\
\text { yet fully functional, and } \\
\text { end of summer when the } \\
\text { effect of heat is stronger }\end{array}$ \\
\hline
\end{tabular}


Table 2. Cont

\begin{tabular}{|c|c|c|c|c|c|c|c|}
\hline Author and Year & Country & Objectives & Subjects & Study Design & Intervention or Factors & Outcomes & Recommendations \\
\hline $\begin{array}{l}\text { Benmarhnia } \\
\text { et al. } 2016[53]\end{array}$ & \multirow{3}{*}{ America/Canada } & $\begin{array}{l}\text { Investigating causal } \\
\text { effects heat action } \\
\text { plans (HAP) and } \\
\text { association with } \\
\text { different subgroups }\end{array}$ & $\begin{array}{l}\text { Male vs. Female; } \\
\geq 65 \text { vs. }<65 ; \\
\text { Education first vs. } \\
\quad \text { third tertile }\end{array}$ & $\begin{array}{l}\text { Quasi-experimental } \\
\text { retrospective - } \\
\text { difference-in-differen } \\
\quad \text { approach }\end{array}$ & $\begin{array}{c}\text { Comparing daily } \\
\text { mortality rates } \\
\text { nc(3000-2003) and post-HAP } \\
\text { introduction (2004-2007) }\end{array}$ & $\begin{array}{c}\text { A reduction in } 2.52 \text { deaths per day } \\
\text { overall with } 2.44 \text { deaths per day } \\
\text { less for older people } \geq 65 \text {; } 2.48 \\
\text { deaths per day less for low SES } \\
\text { group; No differences between } \\
\text { genders }\end{array}$ & $\begin{array}{l}\text { Specifically targeting } \\
\text { vulnerable population } \\
\text { may reduce inequalities } \\
\text { between populations } \\
\text { More frequent home visits } \\
\text { and daily phone calls to } \\
\text { more at-risk individuals }\end{array}$ \\
\hline $\begin{array}{c}\text { Sheridan } 2007 \\
\text { [54] }\end{array}$ & & $\begin{array}{l}\text { Investigate efficacy - } \\
\text { four heat warning } \\
\text { systems in Dayton, } \\
\text { Philadelphia Phoenix, } \\
\text { Arizona, Toronto }\end{array}$ & $\mathrm{n}=908 \geq 65$ & $\begin{array}{l}\text { Cross-sectional } \\
\text { telephone survey }\end{array}$ & $\begin{array}{l}\text { Perception of own } \\
\text { vulnerability, knowledge } \\
\text { of prevention behaviour } \\
\text { and course of action } \\
\text { during heatwaves }\end{array}$ & $\begin{array}{l}\text { Most aware of heat warnings but } \\
\text { few understood what to do; Only } \\
\text { half changed behaviour; Main } \\
\text { source of warnings from television } \\
\text { and radio }\end{array}$ & $\begin{array}{l}\text { Broadcast specific/easy to } \\
\text { understand heat health } \\
\text { advisories } \\
\text { Address warnings } \\
\text { 'blocking out'/confusion } \\
\text { Explain safe use of electric } \\
\text { fans }\end{array}$ \\
\hline $\begin{array}{l}\text { White-Newsome } \\
\text { et al. } 2011 \text { [55] }\end{array}$ & & $\begin{array}{l}\text { Investigate behaviours } \\
\text { and adaptability to } \\
\text { increased indoor } \\
\text { temperatures and } \\
\text { environment in } \\
\text { Detroit }\end{array}$ & $\mathrm{n}=29$ Aged $>65$ & $\begin{array}{l}\text { Cross-sectional } \\
\text { survey of } \\
\text { volunteered } \\
\text { residents }\end{array}$ & $\begin{array}{l}\text { Data collection via hourly } \\
\text { activity logs of eight } \\
\text { heat-adaptive behaviours }\end{array}$ & $\begin{array}{c}\text { Indoor temperature significantly } \\
\text { influenced behaviour; More } \\
\text { adaptive behaviours in high-rises } \\
\text { and highly impervious areas; } \\
\text { Changing clothes, taking additional } \\
\text { showers and going outside rarely } \\
\text { used }\end{array}$ & $\begin{array}{l}\text { Public health } \\
\text { interventions outreach to } \\
\text { this vulnerable group to } \\
\text { encourage full range of } \\
\text { prevention behaviours }\end{array}$ \\
\hline $\begin{array}{l}\text { Abrahamson } \\
\text { et al. } 2009 \text { [56] }\end{array}$ & \multirow[t]{2}{*}{ UK } & $\begin{array}{c}\text { Explore frontline } \\
\text { healthcare } \\
\text { professionals' risks } \\
\text { awareness and } \\
\text { support for older } \\
\text { people at risk of } \\
\text { heatwaves adverse } \\
\text { effects and perceived } \\
\text { barriers to effective } \\
\text { implementation of } \\
\text { HAP }\end{array}$ & $\begin{array}{c}\mathrm{n}=109 \text { covering } \\
\text { three different } \\
\text { socio-economic } \\
\text { areas }\end{array}$ & $\begin{array}{l}\text { Semi-structured } \\
\text { interviews and } \\
\text { focus groups }\end{array}$ & $\begin{array}{l}\text { Awareness of details of } \\
\text { HAP; opinions of self and } \\
\text { organizations' ability to } \\
\text { identify and prioritize } \\
\text { high-risk individuals; } \\
\text { barriers and facilitators to } \\
\text { effective implementation } \\
\text { of HAP }\end{array}$ & $\begin{array}{l}\text { Poor awareness of HAP from } \\
\text { health professionals; Summer } \\
\text { workloads not prioritised with } \\
\text { older people in mind citing } \\
\text { complexities and classification of } \\
\text { vulnerability and infrequency of } \\
\text { heatwaves as barriers }\end{array}$ & $\begin{array}{l}\text { Multidisciplinary } \\
\text { approach to interventions } \\
\text { recommended } \\
\text { Further evaluation of } \\
\text { existing practices }\end{array}$ \\
\hline $\begin{array}{l}\text { Abrahamson } \\
\text { e al. } 2009 \text { [57] }\end{array}$ & & $\begin{array}{c}\text { Investigate } \\
\text { knowledge, } \\
\text { perceptions of heat } \\
\text { health risks, and } \\
\text { protective behaviours } \\
\text { of older people living } \\
\text { independently }\end{array}$ & $\begin{array}{c}\mathrm{n}=73 \\
\text { Aged 72-94 }\end{array}$ & $\begin{array}{l}\text { Semi-structured } \\
\text { interviews }\end{array}$ & $\begin{array}{l}\text { Face-to-face interviews } \\
\text { with subjects } \\
\text { recommended by GPs }\end{array}$ & $\begin{array}{l}\text { Few respondents considered } \\
\text { themselves old or vulnerable or at } \\
\text { risk of heat related illness, despite } \\
\text { being aware of comorbidities; Most } \\
\text { respondents disliked 'nanny state' } \\
\text { approach of intervention }\end{array}$ & $\begin{array}{l}\text { Imbed warnings into } \\
\text { favourite TV programs } \\
\text { Clear/easy to understand } \\
\text { instructions Focus on } \\
\text { most 'at-risk' individuals } \\
\text { by health professionals } \\
\text { Warn community rather } \\
\text { than targeting individuals }\end{array}$ \\
\hline
\end{tabular}


Table 2. Cont

\begin{tabular}{|c|c|c|c|c|c|c|c|}
\hline Author and Year & Country & Objectives & Subjects & Study Design & Intervention or Factors & Outcomes & Recommendations \\
\hline $\begin{array}{c}\text { Wolf et al. } 2010 \\
{[58]}\end{array}$ & & $\begin{array}{l}\text { Investigate older } \\
\text { people self-reported } \\
\text { vulnerability and } \\
\text { subsequent influence } \\
\text { on adaptive behaviour }\end{array}$ & $\begin{array}{l}\mathrm{n}=105 \\
\text { Aged 72-94 in } \\
\text { Norwich and } \\
\quad \text { London }\end{array}$ & $\begin{array}{l}\text { Semi-structured } \\
\text { interviews and } \\
\text { open-ended } \\
\text { questions. } \\
\text { Respondents (A) } \\
\text { and nominated } \\
\text { people (B) to } \\
\text { whom they turned } \\
\text { to for assistance } \\
\text { also interviewed }\end{array}$ & $\begin{array}{c}\text { Perceptions and } \\
\text { knowledge of heat risks } \\
\text { explored including daily } \\
\text { routine, socialisation } \\
\text { habits, physical activity, } \\
\text { actual/hypothetical } \\
\text { behavioural changes in } \\
\text { response to heatwaves, } \\
\text { barriers to do so, medical } \\
\text { conditions and } \\
\text { medications, and type of } \\
\text { housing. }\end{array}$ & $\begin{array}{l}\text { Most (A) did not think they were } \\
\text { vulnerable nor perceive heatwaves } \\
\text { as a threat to themselves; They did } \\
\text { not understand the increased risks } \\
\text { associated with certain medical } \\
\text { conditions and medications; } \\
\text { Reported behaviours more towards } \\
\text { coping rather than mitigation; (B) } \\
\text { respondents displayed inconsistent } \\
\text { and limited knowledge of heat } \\
\text { risks; Also (B) did not want to } \\
\text { impinge on (A) independence; } \\
\text { Potentially exacerbate (A) } \\
\text { vulnerability }\end{array}$ & $\begin{array}{l}\text { Further research into the role } \\
\text { of bonding social capital and } \\
\text { climate change adaptation } \\
\text { Definite need to address } \\
\text { barriers in mitigating } \\
\text { behaviours } \\
\text { Call for government } \\
\text { initiatives to finance local } \\
\text { social development such as } \\
\text { community groups in } \\
\text { providing support thus } \\
\text { empowering the older people } \\
\text { Re-evaluation of adaptation } \\
\text { strategies and policy } \\
\text { effectiveness }\end{array}$ \\
\hline $\begin{array}{l}\text { Herrman et al. } \\
2018 \text { [59] }\end{array}$ & Germany & $\begin{array}{c}\text { Investigate GPs } \\
\text { perceptions on } \\
\text { susceptibility and } \\
\text { nursing care of older } \\
\text { people during } \\
\text { heatwaves in } \\
\text { Baden-Württemberg }\end{array}$ & $\begin{array}{c}n=24 \\
\text { over four districts }\end{array}$ & $\begin{array}{c}\text { Face-to-face } \\
\text { semi-structured } \\
\text { interviews, } \\
\text { Qualitative } \\
\text { software analysed }\end{array}$ & $\begin{array}{l}\text { Exploring knowledge of } \\
\text { heatwaves, perceptions of } \\
\text { older people morbidity } \\
\text { and mortality risks factors } \\
\text { and impact levels of } \\
\text { future climate change to } \\
\text { their well-being }\end{array}$ & $\begin{array}{l}\text { Inconsistent knowledge of } \\
\text { heatwaves amongst GPs; Variable } \\
\text { levels of concern for older people } \\
\text { heat-health based on varied } \\
\text { perceptions of risks; Demonstrable } \\
\text { uncertainties on impact of climate } \\
\text { change on health }\end{array}$ & $\begin{array}{l}\text { More training for GPs on } \\
\text { climate change and } \\
\text { heatwaves impacts on older } \\
\text { people's health } \\
\text { Increase social support and } \\
\text { nursing care for older people } \\
\text { in extreme weather and } \\
\text { heatwaves }\end{array}$ \\
\hline
\end{tabular}




\section{Discussion}

The aim of this review was to gather current evidence on risk factors awareness, perception of vulnerability, and protective behaviours of older people against existing government-issued mitigating measures in the events of heatwaves. This also includes professional and non-professional personnel responsible for the health and welfare of older people. Evidently, several important points are highlighted, including (1) HAPs are heterogeneous, relatively new in development, and lacking formal evaluation; (2) It is difficult to establish causal relationship between HAPs components and outcomes and thus further research and refinement in methodology is required; and (3) Further action is needed to translate knowledge/warnings into heat adaptive behaviours. Consequently, effective and efficient health promotion strategies, involving all sectors of the community is required, transcending organisational and political boundaries [27]. As such, utilization of the Ottawa charter potentially facilitates this process in highlighting gaps and guiding further actions. This framework remains as relevant today as it was first proposed over thirty years ago [28,63].

\subsection{Ottawa Charter Action Areas}

\subsubsection{Build Healthy Public Policy}

National governments have a responsibility to create a more sustainable and healthier world [64]. As evidenced in this study, HAPs were introduced all over the world only after severe losses of lives, coupling with economic losses [65]. It is encouraging to see that inequities are being addressed through implementation of federal and regional initiatives. For example, the LLE program examined by Liotta et al. [50] presents an example of a social policy which bridges the gap between individuals with high social capital and those without. By increasing social support, the program has also reduced health inequity, since those without financial means are able to receive the same care that otherwise only those with higher income can access.

Toloo et al.'s systematic review [66] also found an overall reduction in mortality rates attributable to implementation of HWSs and HAPs, including one study which estimated the total cost of $\$ 210,000$ (USD) to run a HWS versus a much less cost-effective figure of $\$ 4$ million per person in saving 117 lives. Despite this evidence, formal policies and legislations protecting the health of older populations in extreme heat events are still lacking, as identified in this review and reflected in a study in Belgium and The Netherlands [67], especially for socially isolated individuals. Evidently, further action by governments worldwide is urgently required to address this global public health issue as a matter of priority.

\subsubsection{Create Supportive Environments}

The environment in which people live, their income, housing conditions, and access to healthcare all contribute to their ultimate health outcomes [68]. This study has found the same social risk factors that contributed to the mortality/morbidity outcomes during heatwaves $[43,44,58]$. Those living in urban areas in high rise apartments, with highly impervious surfaces and low greenspaces, suffered from the heat island effect [55]. People suffering from chronic diseases and those on medications were the ones most at risk [49]. The aging people with lower income who could not afford air conditioning or running costs were most disadvantaged [43,49], as well as those relying on mobility aids that may or may not be living in appropriate housing conditions [47]. In addition, marginalised people in society, such as those who are homeless, are much worse off since they may not have access to basic amenities or healthcare at all [69].

With an estimate of $68 \%$ of the world population living in urban areas by 2050 and heatwaves posing a serious threat for the urban population, studies have investigated mitigating measures to adapt the built environment, making them more resilient to heatwaves [70-73]. A cool retrofitting toolkit proposed by Hatvani-Kovacs and Boland [71] to remodel existing precincts could make them more energy efficient and affordable, while Bennetts et al. [70] proposed a combination of behavior 
modification and cooling refuges to increase thermal comfort and shielding penetration of heat. Both studies have concluded that there are means to reduce the impact of heatwaves without resorting to excessive energy consumption or high costs $[70,71]$. Those strategies present alternatives to current practices that policy makers could implement to mitigate the impacts of future heatwaves that are sustainable and equitable for older populations and do not further exacerbate greenhouse gas emissions.

\subsubsection{Strengthen Community Actions}

Poor social capital from infrequent social outings and contacts has been acknowledged as a risk factor for older people during heatwaves [48]. Empowerment of communities leads to development of strong social capital which would lend support to individuals in need when they need it most. For example, preventative measures during heatwaves would not have operated as well or as efficiently if not for the efforts of the 'Minsei-iin committees' or the 'buddy systems' described by Takahashi et al. [60] and Sheridan [54]. Further, the importance of recognised NGOs such as red cross cannot be ignored during emergencies [48]. Additional contacts from neighbours, family, and friends also provide essential linkages to improve favourable health outcomes.

However, social contacts and support systems need to be 'active' rather than 'passive', as described by Wolf et al. [58]. Since older people value their independence and nominated support people are reluctant to impinge on their independence, it is of paramount importance that any preventative measures be implemented in a timely ongoing manner to ensure maximal efficiency and effectiveness $[57,58]$.

Furthermore, studies into community cohesion and healthy aging have found that older people are more likely to participate in health promotion initiatives, improved mental well-being and self-evaluated health if they perceived the neighbourhood to be safe and accessible [74]. Coll-Planas et al.'s [75] study into prevention of social isolation and loneliness in the older people through weekly group activities found that after two years, 39.5\% still participated in the activities, almost half maintained contact with each other, and overall they reported improvement in mood and social wellbeing. Similarly, Harada et al.'s study [76], led by Kobe University, found that older people's social network improved significantly following a year-long, 18 theme-based programs aimed at promoting social interactions. It is the duty of local governments and communities to provide older people with opportunities to build upon their social capital, thus lowering the risk associated with extreme heat events.

\subsubsection{Develop Personal Skills}

This study has identified different means in which authorities strived to educate their citizens on preventative measures during heatwaves. The two most commonly utilised methods were via television and radio [57]. However, more modern means of communication have also been employed with some degree of success, such as mobile messaging and social media advisories [44]. Evidently, there is much room for improvement in this area however, as approximately $47 \%$ of respondents still ignored warning messages [47]. Another issue of concern is confusion or poor recall of exact course of actions and under-utilisation of recommended mitigation activities [54,55].

Baker et al.'s study [77] into health literacy and mortality in older people found that poor health literacy is correlated with poor uptake of preventative services, with an overall strongest correlation between reading fluency and all-cause mortality. Geboers et al.'s study [78] also found the same correlation between low education level and low health literacy. Moving beyond just reading or numeracy skills, Serper et al. [79] noted that for older people, cognitive processing such as memory, processing speed, and inductive reasoning all influence functional health. This could perhaps explain the low rates of heat warnings recall, confusion, and ignorance regarding risks to health and lack of translation to protective behaviours $[47,55,57]$. Another interesting point to note is the success of Nitschke et al.'s randomised controlled trial in which multiple 'reminders', such as laminated cards and fridge magnets, were used to facilitate adaptive behaviours [49]. 


\subsubsection{Reorient Health Services}

The evidence gathered in this study highlights the fact that community frontline healthcare services require a more proactive multidisciplinary approach in caring for the older people during heatwaves $[45,46,56,59]$. Johansson et al.'s study [80] in Sweden found that while over $90 \%$ of health professionals have a positive attitude to health promotion within healthcare delivery, in practice there were barriers to implementation. The three most cited barriers to health promotion were large workload, inadequate guidelines, and inexplicit objectives. These barriers echoed the findings of McInnes and Ibrahim's study [46] which concluded that despite the capacity to promote health during heatwaves, extra resources, coordination, and leadership is required.

However, reorientation of healthcare services is possible, as demonstrated by the "The Core Skills in Health Promotion Project' described by Yeatman and Nove [81]—using the capacity building framework and three key elements of partnership, leadership, and commitment to achieve workforce expansion, reallocation of resources, and organizational change. Another way to reorient health services is through modern wearable technology. The global aging population means an increasing need for more cost-effective, efficient early interventions of chronic diseases [82]. Research on wearable, non-invasive, remote monitoring devices, such as fabric-integrated sensors and removable wrist/body sensors, have predicted much promise into a more integrated, sustainable, and equitable healthcare system [83-85]. Older people are more likely to agree to installation of remote monitoring systems in their homes if it means they could continue to live independently for as long as possible, rather than moving to an aged care facility $[86,87]$.

Only by empowering older people with the means to monitor their own health and by facilitating collaboration between them and healthcare providers can we move away from curative services into more preventative and sustainable healthcare systems.

\subsubsection{Interrelatedness of Action Areas}

All five action areas of the Ottawa charter are connected to one another. Optimal health can only be achieved by combining all aspects of social determinants of health and addressing all intrinsic and extrinsic factors influencing health outcomes. A study by Williams et al. [88] has found a similar network of relationship in factors influencing adaptation to extreme heat (see Figure 2). However, only two studies [50,60] demonstrated utilisation of all action areas of the Ottawa charter with seemingly more favourable outcomes (see Table 3).

Evidently, relying on just one or two action areas of the Ottawa charter is not enough. As aforementioned, older people, support personnel, and frontline healthcare professionals all tend to underestimate the risks and level of vulnerability, similar to findings from a previous study by Bittner and Stobel [89]. Furthermore, a recent review by Wondmagegn et al. [90] has identified a substantial burden on healthcare systems in economic terms attributable to extreme heat exposure. Also, consistent with this review, those most affected were older people, females, as well as those from ethnic minorities and with low socio-economic status [90]. Hence, this reinforces the need for a more comprehensive multi-faceted strategy to derive the most effective solutions in solving this complex, but potentially solvable public health problem. 
Table 3. Ottawa charter action areas.

\begin{tabular}{|c|c|c|c|c|c|}
\hline Author/Year & $\begin{array}{c}\text { Build Healthy Public } \\
\text { Policy }\end{array}$ & $\begin{array}{l}\text { Create Supportive } \\
\text { Environment }\end{array}$ & $\begin{array}{c}\text { Strengthen Community } \\
\text { Action }\end{array}$ & Develop Personal Skills & Reorient Health Services \\
\hline Hansen et al. 2011 [43] & N/A & N/A & $\mathrm{N} / \mathrm{A}$ & N/A & $\begin{array}{l}\text { Surveyed healthcare providers and } \\
\text { legislators perceptions and knowledge }\end{array}$ \\
\hline Hansen et al. 2015 [44] & N/A & N/A & N/A & $\begin{array}{l}\text { Surveyed heat health } \\
\text { behaviours }\end{array}$ & N/A \\
\hline Ibrahim et al. 2012 [45] & $\mathrm{N} / \mathrm{A}$ & N/A & N/A & N/A & $\begin{array}{l}\text { Investigated health providers } \\
\text { knowledge and practices }\end{array}$ \\
\hline McInnes et al. 2010 [46] & N/A & N/A & $\mathrm{N} / \mathrm{A}$ & N/A & $\begin{array}{l}\text { Investigated community } \\
\text { healthcare providers roles }\end{array}$ \\
\hline Nitschke et al. 2013 [47] & N/A & N/A & $\mathrm{N} / \mathrm{A}$ & $\begin{array}{l}\text { Surveyed risk factors and } \\
\text { Protective behaviours }\end{array}$ & $\mathrm{N} / \mathrm{A}$ \\
\hline Nitschke et al. 2016 [48] & Evaluation of HWS & $\mathrm{N} / \mathrm{A}$ & $\mathrm{N} / \mathrm{A}$ & $\mathrm{N} / \mathrm{A}$ & $\mathrm{N} / \mathrm{A}$ \\
\hline Nitschke et al. 2017 [49] & Regional policy in place & N/A & $\mathrm{N} / \mathrm{A}$ & $\begin{array}{c}\text { Surveyed protective } \\
\text { behaviours }\end{array}$ & N/A \\
\hline Liotta et al. 2018 [50] & $\begin{array}{l}\text { National and regional } \\
\text { policies in place }\end{array}$ & $\begin{array}{c}\text { Coordinated care } \\
\text { network }\end{array}$ & $\begin{array}{l}\text { Carers and volunteers } \\
\text { involved }\end{array}$ & $\begin{array}{c}\text { Individuals consent } \\
\text { sought }\end{array}$ & Centralised database \\
\hline Michelozzi et al. 2010 [51] & $\begin{array}{l}\text { Evaluated national and } \\
\text { regional policies }\end{array}$ & N/A & $\mathrm{N} / \mathrm{A}$ & N/A & N/A \\
\hline Schifano et al. 2012 [52] & $\begin{array}{c}\text { Evaluated efficacy of } \\
\text { HAPs }\end{array}$ & N/A & N/A & $\mathrm{N} / \mathrm{A}$ & $\mathrm{N} / \mathrm{A}$ \\
\hline Benmarhnia et al. 2016 [53] & $\begin{array}{l}\text { Evaluated effectiveness } \\
\text { of HAP }\end{array}$ & N/A & $\mathrm{N} / \mathrm{A}$ & $\mathrm{N} / \mathrm{A}$ & N/A \\
\hline Sheridan 2007 [54] & $\begin{array}{c}\text { Reviewed HWS } \\
\text { effectiveness }\end{array}$ & N/A & N/A & $\begin{array}{l}\text { Surveyed individual } \\
\text { responses }\end{array}$ & N/A \\
\hline White-Newsome et al. 2011 [55] & $\mathrm{N} / \mathrm{A}$ & $\begin{array}{c}\text { Assess effects of } \\
\text { indoor temperature }\end{array}$ & $\mathrm{N} / \mathrm{A}$ & $\begin{array}{c}\text { Surveyed protective } \\
\text { behaviours }\end{array}$ & N/A \\
\hline Abrahamson et al. 2009 [57] & N/A & N/A & N/A & N/A & $\begin{array}{l}\text { Surveyed health professionals } \\
\text { knowledge and perceptions }\end{array}$ \\
\hline Abrahamson et al. 2009 [56] & N/A & N/A & N/A & $\begin{array}{c}\text { Surveyed awareness and } \\
\text { behaviours }\end{array}$ & N/A \\
\hline Wolf et al. 2010 [58] & N/A & N/A & $\mathrm{N} / \mathrm{A}$ & $\begin{array}{l}\text { Surveyed perceptions of } \\
\text { vulnerability } \\
\text { and behaviours }\end{array}$ & N/A \\
\hline Herrman et al. 2018 [59] & $\mathrm{N} / \mathrm{A}$ & N/A & N/A & $\mathrm{N} / \mathrm{A}$ & $\begin{array}{l}\text { Investigated GPs knowledge } \\
\text { and practices }\end{array}$ \\
\hline Takahashi et al. 2015 [60] & Regional policy in place & $\begin{array}{l}\text { Coordinated local } \\
\text { network }\end{array}$ & $\begin{array}{l}\text { Welfare commissioners } \\
\text { involved }\end{array}$ & $\begin{array}{l}\text { Surveyed prevention } \\
\text { behaviours }\end{array}$ & $\begin{array}{l}\text { Overseen by Institute of } \\
\text { tropical medicine }\end{array}$ \\
\hline
\end{tabular}




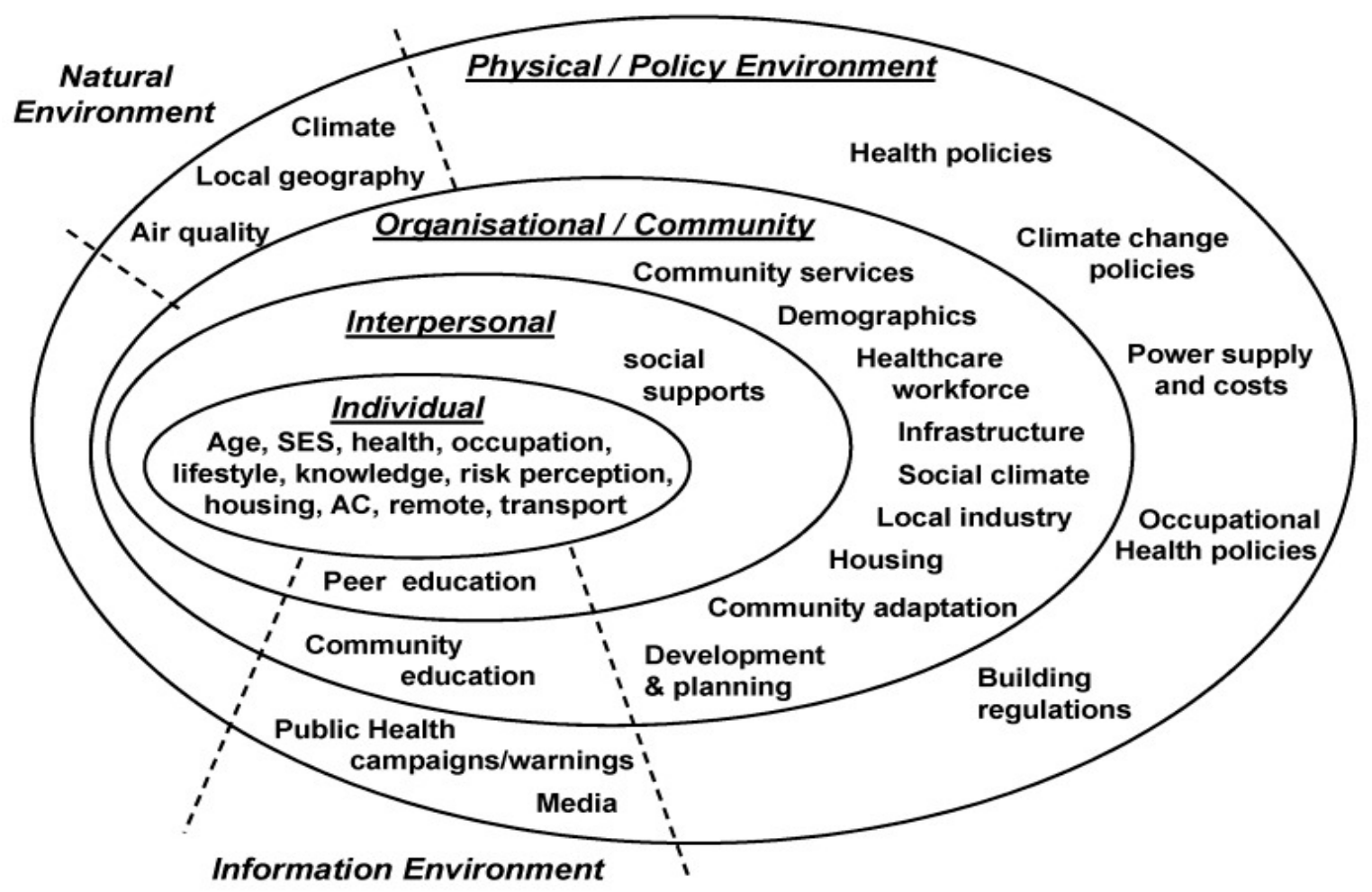

Figure 2. Factors influencing heat adaptation [88].

\subsection{Limitations}

This review focused on only peer-reviewed, English language studies. As such, non-English, unpublished, and grey literature potentially containing relevant informal evaluation of heatwave plans may have been missed. Two studies by Moher et al. [91] and Morrison et al. [92] found no risks of bias from excluding non-English papers. Nevertheless, studies have provided a growing body of evidence that grey literature may provide more detailed evaluation of interventions while not being subjected to publication bias [93,94]. Also, Adams et al. [93] have concluded that results from evaluation of public health interventions may predominantly be held in grey literature, though both studies concluded that the risk of bias is high and replicability of future searches is low when analysing grey literature [93,94].

Though quality assessment of articles in this review was not able to be carried out, individual authors identified their own paper's limitations. These include respondent bias [45,46,55], selection bias, and attrition bias [49,60], comparability issues between data sets [48], recall bias [44,47,57], low response rate [46], inability to generalize outcome to the rest of the population, or not capturing all sections of a community due to no phone access or non-English speakers $[50,54,57]$.

In addition, the adoption of various study designs from 'observational' to 'experimental' means that each study has its own strengths and weaknesses [95]. For example, only two studies $[49,60]$ applied RCT design, which is considered as the 'gold-standard' of experimental designs as it minimizes selection bias, resulting in less confounded outcomes with clearer causalities [95,96]. However, as aforementioned, those studies were not without confounders, as fully randomized or double-blind designs in medical/health research can be fraught with ethical and practical issues [96]. With the exception of quasi-experimental studies [50] and [53] as a 'compromise' from full randomization, the rest of the included studies adopted more observational designs [95]. These study designs are more economical to implement in terms of time and finance but at the same time they are also subject to potential biases, as mentioned by various authors above [95,96]. Moreover, authors of both [95] and [96] have conceded that there is a place and scope for both 'interventional' and 'non-interventional' studies as they can be complementary, depending on the aim and objectives as well as ease of operation, duration, and expenses. 
Overall, there appears to be a gross under-representation of studies from countries with developing economies, as all eighteen articles were from high income developed regions. Yet, studies such as Herold et al. [97]'s have found that each year low income countries have experienced twice the number of hot days compared with high income countries. Notwithstanding the fact that low income countries are responsible for the least portion of global greenhouse gas emissions, they are situated in the most susceptible climatic zones and consequently already suffering disproportionately from global warming compared to developed countries $[97,98]$. This highlights a large gap in research and policy development that requires urgent attention in line with increasing climate change.

\section{Conclusions}

The aim of this review was to identify current measures in mitigating the adverse effects of extreme heat events in older populations. These include self-reported perceptions of vulnerability and risk awareness, as well as social and behavioural factors affecting heat-health outcomes of older people during heatwaves. This study has established that older people remain vulnerable to heat but that heat action plans are making a difference to mortality and morbidity rates overall. Healthcare personnel, both professional and non-professional, have the capacities to effect change, but more dynamic engagement is required. Furthermore, establishing a cause-effect relationship between specific mechanisms of heat action plans and health outcomes remains a challenge. Nevertheless, by analysing the findings against the backdrop of the Ottawa charter, this review highlights the need for a more coordinated approach to solve this potentially recurring public health dilemma. Further research with more rigorous methodology and designs is required in this field. There is a strong need for further research, particularly in low income countries given the projected increase in extreme weather events relating to climate change and the dearth of research evidence on this topic available for inclusion in this review [99]. Finally, there is an urgent need to evaluate existing heat action plans to identify the scope of components, the effectiveness of components and their combination, and adjust or delete redundancies.

Author Contributions: Conceptualization, S.R. and A.V.; methodology, D.P.; investigation and formal analysis, A.V.; resources, S.R.; writing—original draft preparation, A.V.; and writing—review and editing, A.V., S.R, and D.P.

Funding: This research received no external funding.

Acknowledgments: The authors would like to thank the reviewers for their expertise and valuable input.

Conflicts of Interest: The authors declare no conflict of interest.

\section{References}

1. IPCC. 2018: Summary for Policymakers. In Global Warming of $1.5^{\circ} \mathrm{C}$; An IPCC Special Report on the Impacts of Global Warming of $1.5^{\circ} \mathrm{C}$ above Pre-Industrial Levels and Related Global Greenhouse Gas Emission Pathways, in the Context of Strengthening the Global Response to the Threat of Climate Change, Sustainable Development, and Efforts to Eradicate Poverty; Masson-Delmotte, V., Zhai, P., Pörtner, H.O., Roberts, D., Skea, J., Shukla, P.R., Pirani, A., Moufouma-Okia, W., Péan, C., Pidcock, R., et al., Eds.; World Meteorological Organization: Geneva, Switzerland, 2018; p. 32.

2. Semenza, J.C. Climate Change and Human Health. Int. J. Environ. Res. Public Health 2014, 11, 7347-7353. [CrossRef] [PubMed]

3. Semenza, J.C.; Rubin, C.H.; Falter, K.H.; Selanikio, J.D.; Flanders, W.D.; Howe, H.L.; Wilhelm, J.L. Heat-related deaths during the July 1995 heat wave in Chicago. N. Engl. J. Med. 1996, 335, 84-90. [CrossRef]

4. Whitman, S.; Good, G.; Donoghue, E.R.; Benbow, N.; Shou, W.; Mou, S. Mortality in Chicago attributed to the July 1995 heat wave. Am. J. Public Health 1997, 87, 1515-1518. [CrossRef]

5. Fouillet, A.; Rey, G.; Laurent, F.; Pavillon, G.; Bellec, S.; Guihenneuc-Jouyaux, C.; Clavel, J.; Jougla, E.; Hémon, D. Excess mortality related to the August 2003 heat wave in France. Int. Arch. Occup. Environ. Health 2006, 80, 16-24. [CrossRef] 
6. $\quad$ Robine, J.-M.; Cheung, S.L.K.; Le Roy, S.; Van Oyen, H.; Griffiths, C.; Michel, J.-P.; Herrmann, F.R. Death toll exceeded 70,000 in Europe during the summer of 2003. C. R. Biol. 2008, 331, 171-178. [CrossRef]

7. Shaposhnikov, D.; Revich, B.; Bellander, T.; Bedada, G.B.; Bottai, M.; Kharkova, T.; Kvasha, E.; Lezina, E.; Lind, T.; Semutnikova, E.; et al. Mortality Related to Air Pollution with the Moscow Heat Wave and Wildfire of 2010. Epidemiology 2014, 25, 359-364. [CrossRef]

8. Perkins-Kirkpatrick, S.E.; White, C.J.; Alexander, L.V.; Argüeso, D.; Boschat, G.; Cowan, T.; Evans, J.P.; Ekström, M.; Oliver, E.C.J.; Phatak, A.; et al. Natural hazards in Australia: Heatwaves. Clim. Chang. 2016, 139, 101-114. [CrossRef]

9. Oudin Åström, D.; Schifano, P.; Asta, F.; Lallo, A.; Michelozzi, P.; Rocklöv, J.; Forsberg, B. The effect of heat waves on mortality in susceptible groups: A cohort study of a mediterranean and a northern European City. Environ. Health 2015, 14, 30. [CrossRef] [PubMed]

10. Oudin Åström, D.; Bertil, F.; Joacim, R. Heat wave impact on morbidity and mortality in the elderly population: A review of recent studies. Maturitas 2011, 69, 99-105. [CrossRef] [PubMed]

11. Leyva, E.W.A.; Beaman, A.; Davidson, P.M. Health Impact of Climate Change in Older People: An Integrative Review and Implications for Nursing: Climate Change, Ageing, and Nursing. J. Nurs. Scholarsh. 2017, 49, 670-678. [CrossRef]

12. Arbuthnott, K.G.; Hajat, S. The health effects of hotter summers and heat waves in the population of the United Kingdom: A review of the evidence. Environ. Health 2017, 16, 119. [CrossRef]

13. Kenny, G.P.; Yardley, J.; Brown, C.; Sigal, R.J.; Jay, O. Heat stress in older individuals and patients with common chronic diseases. CMAJ 2010, 182, 1053-1060. [CrossRef]

14. Cheng, J.; Xu, Z.; Bambrick, H.; Su, H.; Tong, S.; Hu, W. Heatwave and elderly mortality: An evaluation of death burden and health costs considering short-term mortality displacement. Environ. Int. 2018, 115, 334-342. [CrossRef] [PubMed]

15. Yu, W.; Mengersen, K.; Wang, X.; Ye, X.; Guo, Y.; Pan, X.; Tong, S. Daily average temperature and mortality among the elderly: A meta-analysis and systematic review of epidemiological evidence. Int. J. Biometeorol. 2012, 56, 569-581. [CrossRef]

16. Jeyakumaran, N.; Gabb, G.; Rowett, D.; Tadros, R. Cardiovascular Disease, Medications and Heat: What Precautionary Advice is Available? Heart Lung Circ. 2016, 25, S317. [CrossRef]

17. Bunker, A.; Wildenhain, J.; Vandenbergh, A.; Henschke, N.; Rocklöv, J.; Hajat, S.; Sauerborn, R. Effects of Air Temperature on Climate-Sensitive Mortality and Morbidity Outcomes in the Elderly; a Systematic Review and Meta-analysis of Epidemiological Evidence. EBioMedicine 2016, 6, 258-268. [CrossRef] [PubMed]

18. United Nations, Department of Economic and Social Affairs, Population Division. World Population Ageing 2017—Highlights (ST/ESA/SER.A/397). 2017. Available online: https://www.un.org/en/development/desa/ population/publications/pdf/ageing/WPA2017_Highlights.pdf (accessed on 30 August 2019).

19. United Nations, Department of Economic and Social Affairs, Population Division. World Population Prospects 2019: Highlights (ST/ESA/SER.A/423). 2019. Available online: https://population.un.org/wpp/ Publications/Files/WPP2019_10KeyFindings.pdf (accessed on 30 August 2019).

20. Reid, C.E.; O’Neill, M.S.; Gronlund, C.J.; Brines, S.J.; Brown, D.G.; Diez-Roux, A.V.; Schwartz, J. Mapping community determinants of heat vulnerability. Environ. Health Perspect. 2009, 117, 1730-1736. [CrossRef]

21. Curriero, F.C.; Heiner, K.S.; Samet, J.M.; Zeger, S.L.; Strug, L.; Patz, J.A. Temperature and mortality in 11 cities of the eastern United States. Am. J. Epidemiol. 2002, 155, 80-87. [CrossRef]

22. Kim, Y.; Joh, S. A vulnerability study of the low-income elderly in the context of high temperature and mortality in Seoul, Korea. Sci. Total Environ. 2006, 371, 82-88. [CrossRef]

23. Medina-Ramón, M.; Zanobetti, A.; Cavanagh, D.P.; Schwartz, J. Extreme temperatures and mortality: Assessing effect modification by personal characteristics and specific cause of death in a multi-city case-only analysis. Environ. Health Perspect. 2006, 114, 1331-1336. [CrossRef]

24. O'Neill, M.S.; Zanobetti, A.; Schwartz, J. Disparities by race in heat-related mortality in four US cities: The role of air conditioning prevalence. J. Urban Health 2005, 82, 191-197. [CrossRef]

25. Yang, J.; Yin, P.; Sun, J.; Wang, B.; Zhou, M.; Li, M.; Tong, S.; Meng, B.; Guo, Y.; Liu, Q. Heatwave and mortality in 31 major Chinese cities: Definition, vulnerability and implications. Sci. Total Environ. 2019, 649, 695-702. [CrossRef] 
26. Marmot, M.P.; Friel, S.P.; Bell, R.P.; Houweling, T.A.J.P.; Taylor, S.P.; Commission on Social Determinants of Health. Closing the gap in a generation: Health equity through action on the social determinants of health. Lancet 2008, 372, 1661-1669. [CrossRef]

27. World Health Organization. The Ottawa Charter for Health Promotion. Available online: https://www.who. int/healthpromotion/conferences/previous/ottawa/en/index1.html (accessed on 30 August 2019).

28. Fry, D.; Zask, A. Applying the Ottawa Charter to inform health promotion programme design. Health Promot. Int. 2017, 32, 901-912. [CrossRef] [PubMed]

29. Britto, M.T.; Fuller, S.C.; Kaplan, H.C.; Kotagal, U.; Lannon, C.; Margolis, P.A.; Muething, S.E.; Schoettker, P.J.; Seid, M. Using a network organisational architecture to support the development of Learning Healthcare Systems. BMJ Qual. Saf. 2018, 27, 937-946. [CrossRef] [PubMed]

30. Moher, D.; Liberati, A.; Tetzlaff, J.; Altman, D.G.; Prisma Group. Preferred Reporting Items for Systematic Reviews and Meta-Analyses: The PRISMA Statement. J. Clin. Epidemiol. 2009, 62, 1006-1012. [CrossRef]

31. Fink, R.; Erzen, I.; Medved, S. Symptomatic Response of the Elderly with Cardiovascular Disease during a Heat Wave in Slovenia. Cent. Eur. J. Public Health 2017, 25, 293-298. [CrossRef]

32. Loughnan, M.E.; Carroll, M.; Tapper, N. Learning from our older people: Pilot study findings on responding to heat. Australas. J. Ageing 2014, 33, 271-277. [CrossRef]

33. Hansen, A.; Bi, P.; Nitschke, M.; Pisaniello, D.; Newbury, J.; Kitson, A. Older persons and heat-susceptibility: The role of health promotion in a changing climate. Health Promot. J. Austr. 2011, 22, S17-S20. [CrossRef]

34. Hendry, M.; Williams, N.H.; Wilkinson, C. A survey of local health promotion initiatives for older people in Wales. BMC Public Health 2008, 8, 217. [CrossRef]

35. Nitschke, M.; Tucker, G.R.; Hansen, A.L.; Williams, S.; Zhang, Y.; Bi, P. Impact of two recent extreme heat episodes on morbidity and mortality in Adelaide, South Australia: A case-series analysis. Environ. Health 2011, 10, 42. [CrossRef] [PubMed]

36. Schifano, P.; Cappai, G.; De Sario, M.; Michelozzi, P.; Marino, C.; Bargagli, A.M.; Perucci, C.A. Susceptibility to heat wave-related mortality: A follow-up study of a cohort of elderly in Rome. Environ. Health 2009, 8, 50. [CrossRef]

37. Zhang, Y.; Nitschke, M.; Krackowizer, A.; Dear, K.; Pisaniello, D.; Weinstein, P.; Tucker, G.; Shakib, S.; Bi, P. Risk factors of direct heat-related hospital admissions during the 2009 heatwave in Adelaide, Australia: A matched case-control study. BMJ Open 2016, 6, e010666. [CrossRef]

38. Haque, M.A.; Budi, A.; Azam Malik, A.; Suzanne Yamamoto, S.; Louis, V.R.; Sauerborn, R. Health coping strategies of the people vulnerable to climate change in a resource-poor rural setting in Bangladesh. BMC Public Health 2013, 13, 565. [CrossRef]

39. Khare, S.; Hajat, S.; Kovats, S.; Lefevre, C.E.; de Bruin, W.B.; Dessai, S.; Bone, A. Heat protection behaviour in the UK: Results of an online survey after the 2013 heatwave. BMC Public Health 2015, 15, 878. [CrossRef]

40. Kreslake, J.M.; Price, K.M.; Sarfaty, M. Developing effective communication materials on the health effects of climate change for vulnerable groups: A mixed methods study. BMC Public Health 2016, 16, 946. [CrossRef]

41. Price, K.; Perron, S.; King, N. Implementation of the Montreal Heat Response Plan during the 2010 Heat Wave. Can. J. Public Health 2013, 104, E96-E100. [CrossRef] [PubMed]

42. Price, K.; Benmarhnia, T.; Gaudet, J.; Kaiser, D.; Sadoine, M.L.; Perron, S.; Smargiassi, A. The Montreal heat response plan: Evaluation of its implementation towards healthcare professionals and vulnerable populations. Can. J. Public Health 2018, 109, 108-116. [CrossRef]

43. Hansen, A.; Bi, P.; Nitschke, M.; Pisaniello, D.; Newbury, J.; Kitson, A. Perceptions of Heat-Susceptibility in Older Persons: Barriers to Adaptation. Int. J. Environ. Res. Public Health 2011, 8, 4714-4728. [CrossRef] [PubMed]

44. Hansen, A.; Peng, B.; Pisaniello, D.; Nitschke, M.; Tucker, G.; Newbury, J.; Kitson, A.; Dal Grande, E.; Avery, J.; Ying, Z.; et al. Heat-health behaviours of older people in two Australian states. Australas. J. Ageing 2015, 34, E19-E25. [CrossRef]

45. Ibrahim, J.E.; McInnes, J.A.; Andrianopoulos, N.; Evans, S. Minimising harm from heatwaves: A survey of awareness, knowledge, and practices of health professionals and care providers in Victoria, Australia. Int. J. Public Health 2012, 57, 297-304. [CrossRef]

46. McInnes, J.A.; Ibrahim, J.E. Minimising harm to older Victorians from heatwaves: A qualitative study of the role of community-based health profession and carer organisations. Australas. J. Ageing 2010, 29, 104-110. [CrossRef] 
47. Nitschke, M.; Hansen, A.; Bi, P.; Pisaniello, D.; Newbury, J.; Kitson, A.; Tucker, G.; Avery, J.; Dal Grande, E. Risk Factors, Health Effects and Behaviour in Older People during Extreme Heat: A Survey in South Australia. Int. J. Environ. Res. Public Health 2013, 10, 6721-6733. [CrossRef]

48. Nitschke, M.; Tucker, G.; Hansen, A.; Williams, S.; Zhang, Y.; Bi, P. Evaluation of a heat warning system in Adelaide, South Australia, using case-series analysis. BMJ Open 2016, 6, e012125. [CrossRef]

49. Nitschke, M.; Krackowizer, A.; Hansen, A.L.; Bi, P.; Tucker, G.R. Heat health messages: A randomized controlled trial of a preventative messages tool in the older population of South Australia. Int. J. Environ. Res. Public Health 2017, 14, 992. [CrossRef] [PubMed]

50. Liotta, G.; Inzerilli, M.C.; Palombi, L.; Madaro, O.; Orlando, S.; Scarcella, P.; Betti, D.; Marazzi, M.C. Social Interventions to Prevent Heat-Related Mortality in the Older Adult in Rome, Italy: A Quasi-Experimental Study. Int. J. Environ. Res. Public Health 2018, 15, 715. [CrossRef] [PubMed]

51. Michelozzi, P.; de'Donato, F.K.; Bargagli, A.M.; D’Ippoliti, D.; De Sario, M.; Marino, C.; Schifano, P.; Cappai, G.; Leone, M.; Kirchmayer, U.; et al. Surveillance of summer mortality and preparedness to reduce the health impact of heat waves in Italy. Int. J. Environ. Res. Public Health 2010, 7, 2256-2273. [CrossRef]

52. Schifano, P.; Leone, M.; De Sario, M.; de’Donato, F.; Bargagli, A.M.; D’Ippoliti, D.; Marino, C.; Michelozzi, P. Changes in the effects of heat on mortality among the elderly from 1998-2010: Results from a multicenter time series study in Italy. Environ. Health 2012, 11, 58. [CrossRef]

53. Benmarhnia, T.; Bailey, Z.; Kaiser, D.; Auger, N.; King, N.; Kaufman, J.S. A Difference-in-Differences Approach to Assess the Effect of a Heat Action Plan on Heat-Related Mortality, and Differences in Effectiveness According to Sex, Age, and Socioeconomic Status (Montreal, Quebec). Environ. Health Perspect. 2016, 124, 1694-1699. [CrossRef] [PubMed]

54. Sheridan, S.C. A survey of public perception and response to heat warnings across four North American cities: An evaluation of municipal effectiveness. Int. J. Biometeorol. 2007, 52, 3-15. [CrossRef]

55. White-Newsome, J.L.; Sanchez, B.N.; Parker, E.A.; Dvonch, J.T.; Zhang, Z.Z.; O’Neill, M.S. Assessing heat-adaptive behaviors among older, urban-dwelling adults. Maturitas 2011, 70, 85-91. [CrossRef]

56. Abrahamson, V.; Raine, R. Health and social care responses to the Department of Health Heatwave Plan. J. Public Health 2009, 31, 478-489. [CrossRef] [PubMed]

57. Abrahamson, V.; Wolf, J.; Lorenzoni, I.; Fenn, B.; Kovats, S.; Wilkinson, P.; Adger, W.N.; Raine, R. Perceptions of heatwave risks to health: Interview-based study of older people in London and Norwich, UK. J. Public Health 2009, 31, 119-126. [CrossRef]

58. Wolf, J.; Adger, W.N.; Lorenzoni, I.; Abrahamson, V.; Raine, R. Social capital, individual responses to heat waves and climate change adaptation: An empirical study of two UK cities. Glob. Environ. Chang. 2010, 20, 44-52. [CrossRef]

59. Herrmann, A.; Sauerborn, R. General Practitioners' Perceptions of Heat Health Impacts on the Elderly in the Face of Climate Change-A Qualitative Study in Baden-Wurttemberg, Germany. Int. J. Environ. Res. Public Health 2018, 15, 843. [CrossRef]

60. Takahashi, N.; Nakao, R.; Ueda, K.; Ono, M.; Kondo, M.; Honda, Y.; Hashizume, M. Community trial on heat related-illness prevention behaviors and knowledge for the elderly. Int. J. Environ. Res. Public Health 2015, 12, 3188-3214. [CrossRef]

61. German Federal Ministry for the Environment, Nature Conservation, Building and Nuclear Safety. Recommendations for Action Heat Action Plans to Protect Human Health. Available online: https://www. bmu.de/fileadmin/Daten_BMU/Download_PDF/Klimaschutz/hap_handlungsempfehlungen_en_bf.pdf (accessed on 30 August 2019).

62. Heatwave Plan for Victoria. Available online: http://docs2.health.vic.gov.au/docs/doc/ 45C7A59BDE5B1E95CA257A360015AAB5/\%24FILE/Heatwave\%20plan\%20for\%20Victoria.pdf (accessed on 30 August 2019).

63. Spencer, G.; Corbin, J.H.; Miedema, E. Sustainable development goals for health promotion: A critical frame analysis. Health Promot. Int. 2019, 34, 847-858. [CrossRef]

64. Labonté, R. Health promotion in an age of normative equity and rampant inequality. Int. J. Health Policy 2016, 5, 675-682. [CrossRef] [PubMed]

65. Martinez, G.S.; Imai, C.; Masumo, K. Local heat stroke prevention plans in Japan: Characteristics and elements for public health adaptation to climate change. Int. J. Environ. Res. Public Health 2011, 8, 4563-4581. [CrossRef] 
66. Toloo, G.; FitzGerald, G.; Aitken, P.; Verrall, K.; Tong, S. Evaluating the effectiveness of heat warning systems: Systematic review of epidemiological evidence. Int. J. Public Health 2013, 58, 667-681. [CrossRef]

67. Van Loenhout, J.A.; Roiguez-Llanes, J.M.; Guha-Sapir, D. Stakeholders' Perception on National Heatwave Plans and Their Local Implementation in Belgium and The Netherlands. Int. J. Environ. Res. Public Health 2016, 13, 1120. [CrossRef] [PubMed]

68. Marmot, M.; Bell, R. Fair society, healthy lives. Public Health 2012, 126, S4-S10. [CrossRef]

69. Bassil, K.L.; Cole, D.C. Effectiveness of public health interventions in reducing morbidity and mortality during heat episodes: A structured review. Int. J. Environ. Res. Public Health 2010, 7, 991-1001. [CrossRef]

70. Bennetts, H.; Pullen, S.; Zillante, G. Design strategies for houses subject to heatwaves. Open House Int. 2012, 37, 29-38.

71. Hatvani-Kovacs, G.; Boland, J. Retrofitting Precincts for Heatwave Resilience: Challenges and Barriers in Australian Context. Challenges 2015, 6, 3-25. [CrossRef]

72. Keramitsoglou, I.; Sismanidis, P.; Analitis, A.; Butler, T.; Founda, D.; Giannakopoulos, C.; Giannatou, E.; Karali, A.; Katsouyanni, K.; Kendrovski, V.; et al. Urban thermal risk reduction: Developing and implementing spatially explicit services for resilient cities. Sustain. Cities Soc. 2017, 34, 56-68. [CrossRef]

73. United Nations, Department of Economic and Social Affairs, Population Division. World Urbanization Prospects 2018: Highlights (ST/ESA/SER.A/421). 2019. Available online: https://population.un.org/wup/ Publications/Files/WUP2018-Highlights.pdf (accessed on 1 September 2019).

74. Greenfield, E.A. Healthy Aging and Age-Friendly Community Initiatives. Public Policy Aging Rep. 2015, 25, 43-46. [CrossRef]

75. Coll-Planas, L.; Valle Gómez, G.; Bonilla, P.; Masat, T.; Puig, T.; Monteserin, R. Promoting social capital to alleviate loneliness and improve health among older people in Spain. Health Soc. Care Community 2017, 25, 145-157. [CrossRef]

76. Harada, K.; Masumoto, K.; Katagiri, K.; Fukuzawa, A.; Chogahara, M.; Kondo, N.; Okada, S. Community intervention to increase neighborhood social network among Japanese older adults. Geriatr. Gerontol. Int. 2018, 18, 462-469. [CrossRef]

77. Baker, D.W.; Wolf, M.S.; Feinglass, J.; Thompson, J.A.; Gazmararian, J.A.; Huang, J. Health Literacy and Mortality Among Elderly Persons. Arch. Intern. Med. 2007, 167, 1503-1509. [CrossRef]

78. Geboers, B.; de Winter, A.F.; Spoorenberg, S.L.W.; Wynia, K.; Reijneveld, S.A. The association between health literacy and self-management abilities in adults aged 75 and older, and its moderators. Qual. Life Res. 2016, 25, 2869-2877. [CrossRef] [PubMed]

79. Serper, M.; Patzer, R.E.; Curtis, L.M.; Smith, S.G.; O'Conor, R.; Baker, D.W.; Wolf, M.S. Health Literacy, Cognitive Ability, and Functional Health Status among Older Adults. Health Serv. Res. 2014, 49, 1249-1267. [CrossRef] [PubMed]

80. Johansson, H.; Stenlund, H.; Lundström, L.; Weinehall, L. Reorientation to more health promotion in health services-A study of barriers and possibilities from the perspective of health professionals. J. Multidiscip. Health 2010, 3, 213-224. [CrossRef] [PubMed]

81. Yeatman, H.R.; Nove, T. Reorienting health services with capacity building: A case study of the Core Skills in Health Promotion Project. Health Promot. Int. 2002, 17, 341-350. [CrossRef]

82. Baig, M.M.; GholamHosseini, H.; Moqeem, A.A.; Mirza, F.; Lindén, M. A Systematic Review of Wearable Patient Monitoring Systems-Current Challenges and Opportunities for Clinical Adoption. J. Med. Syst. 2017, 41, 115. [CrossRef]

83. Majumder, S.; Mondal, T.; Deen, M.J. Wearable sensors for remote health monitoring. Sensors 2017, 17, 130. [CrossRef]

84. Munos, B.; Baker, P.C.; Bot, B.M.; Crouthamel, M.; de Vries, G.; Ferguson, I.; Hixson, J.D.; Malek, L.A.; Mastrototaro, J.J.; Misra, V.; et al. Mobile health: The power of wearables, sensors, and apps to transform clinical trials: Mobile technology and clinical trials. Ann. N. Y. Acad. Sci. 2016, 1375, 3-18. [CrossRef]

85. Wagner, F.; Basran, J.; Dal Bello-Haas, V. A review of monitoring technology for use with older adults. J. Geriatr. Phys. Ther. 2012, 35, 28-34. [CrossRef]

86. Pol, M.; van Nes, F.; van Hartingsveldt, M.; Buurman, B.; de Rooij, S.; Kröse, B. Older People's Perspectives Regarding the Use of Sensor Monitoring in Their Home. Gerontologist 2016, 56, 485-493. [CrossRef] 
87. Claes, V.; Devriendt, E.; Tournoy, J.; Milisen, K. Attitudes and perceptions of adults of 60 years and older towards in-home monitoring of the activities of daily living with contactless sensors: An explorative study. Int. J. Nurs. Stud. 2015, 52, 134-148. [CrossRef]

88. Williams, S.; Bi, P.; Newbury, J.; Robinson, G.; Pisaniello, D.; Saniotis, A.; Hansen, A. Extreme heat and health: Perspectives from health service providers in rural and remote communities in South Australia. Int. J. Environ. Res. Public Health 2013, 10, 5565-5583. [CrossRef] [PubMed]

89. Bittner, M.-I.; Stößel, U. Perceptions of heatwave risks to health: Results of an qualitative interview study with older people and their carers in Freiburg, Germany. Psycho-Soc. Med. 2012, 9, Doc05.

90. Wondmagegn, B.Y.; Xiang, J.; Williams, S.; Pisaniello, D.; Bi, P. What do we know about the healthcare costs of extreme heat exposure? A comprehensive literature review. Sci. Total Environ. 2019, 657, 608-618. [CrossRef] [PubMed]

91. Moher, D.; Pham; Klassen, T.P.; Schulz, K.F.; Berlin, J.A.; Jadad, A.R.; Liberati, A. What contributions do languages other than English make on the results of meta-analyses? J. Clin. Epidemiol. 2000, 53, 964-972. [CrossRef]

92. Morrison, A.; Polisena, J.; Husereau, D.; Moulton, K.; Clark, M.; Fiander, M.; Mierzwinski-Urban, M.; Clifford, T.; Hutton, B.; Rabb, D. The effect of english-language restriction on systematic review-based meta-analyses: A systematic review of empirical studies. Int. J. Technol. Assess. Health Care 2012, 28, 138-144. [CrossRef]

93. Adams, J.; Hillier-Brown, F.C.; Moore, H.J.; Lake, A.A.; Araujo-Soares, V.; White, M.; Summerbell, C. Searching and synthesising 'grey literature' and 'grey information' in public health: Critical reflections on three case studies. Syst. Rev. 2016, 5, 164. [CrossRef]

94. Mahood, Q.; Eerd, D.V.; Irvin, E. Searching for grey literature for systematic reviews: Challenges and benefits. Res. Synth. Methods 2014, 5, 221-234. [CrossRef]

95. Ahmad, I.; Marwat, M.; Khan, H. Comparative analysis of study designs in health research. Gomal J. Med. Sci. 2014, 11, 242-244.

96. Jehan, F.; Kazi, A.M. Basic study designs in health research. J. Pak. Med. Assoc. 2012, 62, 408-411.

97. Herold, N.; Alexander, L.; Green, D.; Donat, M. Greater increases in temperature extremes in low versus high income countries. Environ. Res. Lett. 2017, 12, 34007. [CrossRef]

98. Ahmadalipour, A.; Moradkhani, H.; Kumar, M. Mortality risk from heat stress expected to hit poorest nations the hardest. Clim. Chang. 2019, 152, 569-579. [CrossRef]

99. Campbell, S.; Remenyi, T.A.; White, C.J.; Johnston, F.H. Heatwave and health impact research: A global review. Health Place 2018, 53, 210-218. [CrossRef] [PubMed] 